\title{
Enhanced dispersion of epicardial activation-recovery intervals at sites of histological inhomogeneity during regional cardiac ischaemia and reperfusion
}

\author{
E Gottwald, M Gottwald, S Dhein
}

\begin{abstract}
Objective-To examine how epicardial activation and repolarisation patterns change in the course of ischaemia, and how these changes are related to the underlying histological structures.

Methods-Langendorff perfused isolated rabbit hearts were submitted to $30 \mathrm{~min}$ utes of left anterior descending coronary artery occlusion followed by $\mathbf{3 0}$ minutes of reperfusion. A 256 channel epicardial map was plotted during the various experimental phases. Activation time points were determined as $t\left(d U / d t_{\text {min }}\right)$ and repolarisation time points as $\mathrm{t}\left(\mathrm{dU} / \mathrm{dt}_{\mathrm{max}}\right)$. From these data the local activation-recovery interval (ARI), its dispersion (SD of ARI), and the geometry of the activation spread could be analysed. After the experiments the hearts were processed histologically and the mapping data were projected onto histological slides.
\end{abstract}

Results-There was elevation of the ST segment within the occluded area, which recovered during reperfusion. Within this area, ARI was significantly shortened and its dispersion was maximally enhanced. The enhancement of dispersion was pronounced at sites of histological inhomogeneity like fat, connective tissue, or vessels. There was also a change in the preferential direction of activation spread within the occluded zone with a marked transverse propagation of the activation wavefront, whereas under normal conditions the activation followed the longitudinal fibre axis. In addition, the total activation time in the occluded area was significantly prolonged.

Conclusions-Ischaemia alters the local activation pattern with enhanced dispersion, especially at sites of histological irregularity, transverse shift of the activation waves, and a general slowing of conduction, which may explain the increased susceptibility to arrhythmia in hearts with enhanced histological irregularities-for example, an infarct or in multi-infarcted hearts, or after myocarditis.

(Heart 1998;79:474-480)

Keywords: dispersion; epicardial activation-recovery interval; ischaemia
One of the most striking problems with cardiac ischaemia and reperfusion is the occurrence of severe arrhythmias, especially ventricular fibrillation. Although the underlying pathophysiological mechanisms are heterogeneous, a major role has been attributed to the rise in $\left[\mathrm{K}^{+}\right]_{\mathrm{e}}{ }^{1-3}$ and to rises in $\left[\mathrm{Ca}^{2+}\right]_{\mathrm{i}}$, which seem to be associated with certain types of early afterdepolarisation. ${ }^{4}$ Furthermore, besides initiating the flow of the so called injury current ${ }^{5}$ generated by current sources and sinks, which can also induce arrhythmia, hypoxia leads to an increase in longitudinal resistance, which is believed to be caused by closure of gap junction channels. ${ }^{67}$ This cellular uncoupling may play an important role in the genesis of some forms of reentrant arrhythmia after 15 to 20 minutes of ischaemia (type Ib arrhythmia). ${ }^{8-12}$ This uncoupling is also caused by the rise in $\left[\mathrm{Ca}^{2+}\right] \mathrm{i}^{13}{ }^{14}$ metabolic acidification, ${ }^{15}$ ATP depletion, ${ }^{16} \mathrm{LTB}_{4}$ released from activated leucocytes, ${ }^{8}$ diacylglycerols, ${ }^{17}$ and accumulation of long chain acylcarnitines. ${ }^{18}$ Uncoupling, however, is known to result in an increase in dispersion of action potential duration. ${ }^{19-21}$ From these computer simulation studies it was predicted that dispersion can be enhanced at sites of tissue inhomogeneity-for example non-excitable tissues such as vessels, fat, and connective tissue-owing to inhomogeneous current flow from excited to not yet excited tissue, but not to non-excitable sites, if conduction is slowed or uncoupling occurs. Since such a condition could occur in regional cardiac ischaemia, we tried to determine whether regional ischaemia enhances dispersion, especially in regions of inhomogeneity and at nonexcitable sites.

\section{Methods}

HEART PREPARATION

Male New Zealand White rabbits (Fortkamp, Lengerich, Germany; fed ad libitum, body weight: 2000 to $2500 \mathrm{~g}$ ) were killed by a sharp blow on the neck and subsequently exsanguinated. Blood clotting was prevented by intravenous administration of heparin $(1000 \mathrm{IU} / \mathrm{ml} / \mathrm{kg})$ five minutes before the animals were killed. The heart was transferred to a Langendorff apparatus and perfused at a constant pressure of $70 \mathrm{~cm} \mathrm{H}_{2} \mathrm{O}$ with Tyrode solution (composition (mmol/1): $\mathrm{Na}^{+}$161.02, $\mathrm{K}^{+}$5.36, $\mathrm{Ca}^{2+} 1.8, \mathrm{Mg}^{2+} 1.05, \mathrm{Cl}^{-}$147.86, $\mathrm{HCO}_{3}{ }^{-}$23.8, $\mathrm{PO}_{4}{ }^{2-} 0.42$, glucose 11.1 , equilibrated with $95 \% \quad \mathrm{O}_{2}$ and $5 \% \mathrm{CO}_{2}, 37^{\circ} \mathrm{C}$ ). Left ventricular pressure was measured by an 
A

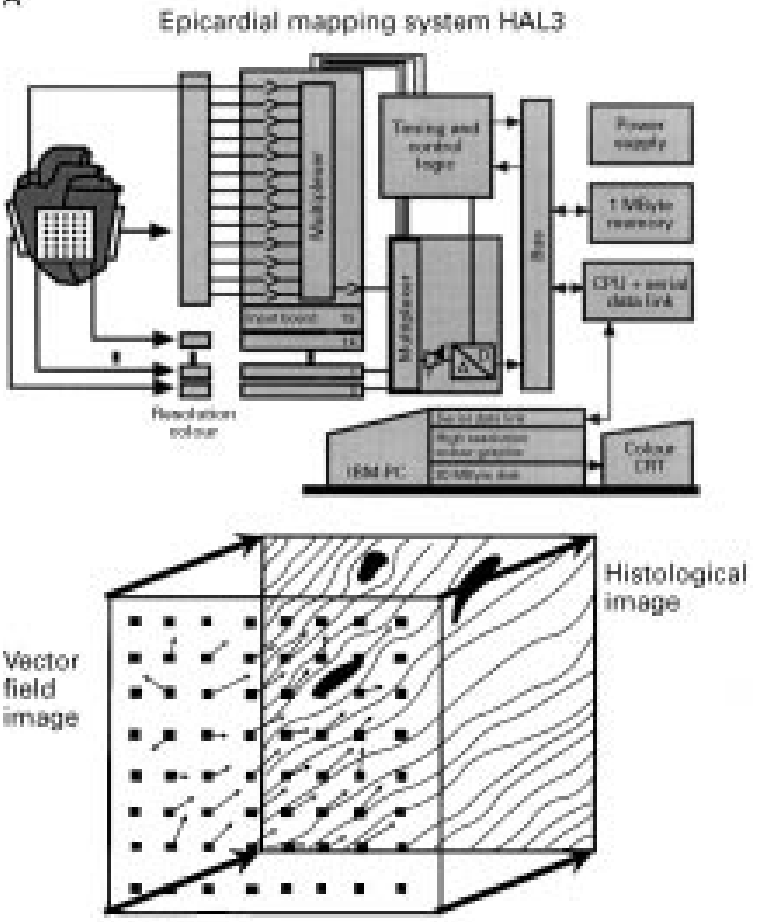

Construction of a voctor

B
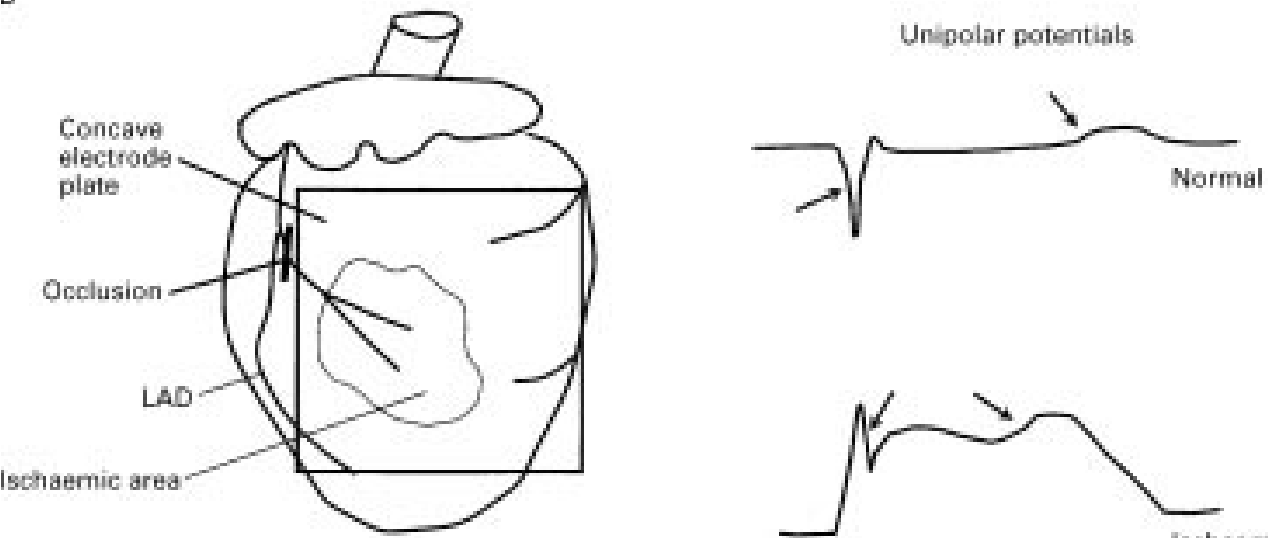

Left ventricular wall
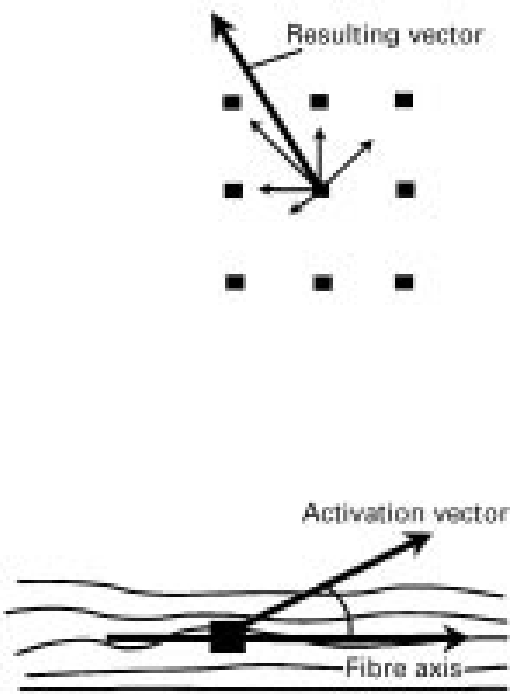

EPICARDIAL POTENTIAL MAPPING

To assess the electrocardiological alterations in the spontaneously beating hearts during the entire experiment, we performed epicardial potential mapping using HAL3, developed in

cooperation with ELSA, Aachen, Germany, providing 256 channels with spatial resolution of $1 \mathrm{~mm}$, temporal resolution $4 \mathrm{kHz} /$ channel, amplitude resolution $0.04 \mathrm{mV}$, and interchannel coupling $<-60 \mathrm{~dB}$ (further details in ${ }^{22-24}$ and fig 1).

Four concave electrode grids consisting of $64 \mathrm{AgCl}$ electrodes arranged in an $8 \times 8$ orthogonal fashion were each attached smoothly to the epicardium. The mapping data were processed for determination of the activation time points and repolarisation time points of each electrode as $\mathrm{t}\left(\mathrm{dU} / \mathrm{dt}_{\text {min }}\right)$ and $\mathrm{t}\left(\mathrm{dU} / \mathrm{dt}_{\max }\right)$, respectively. These time points were calculated by computer and thereafter controlled by the 
A
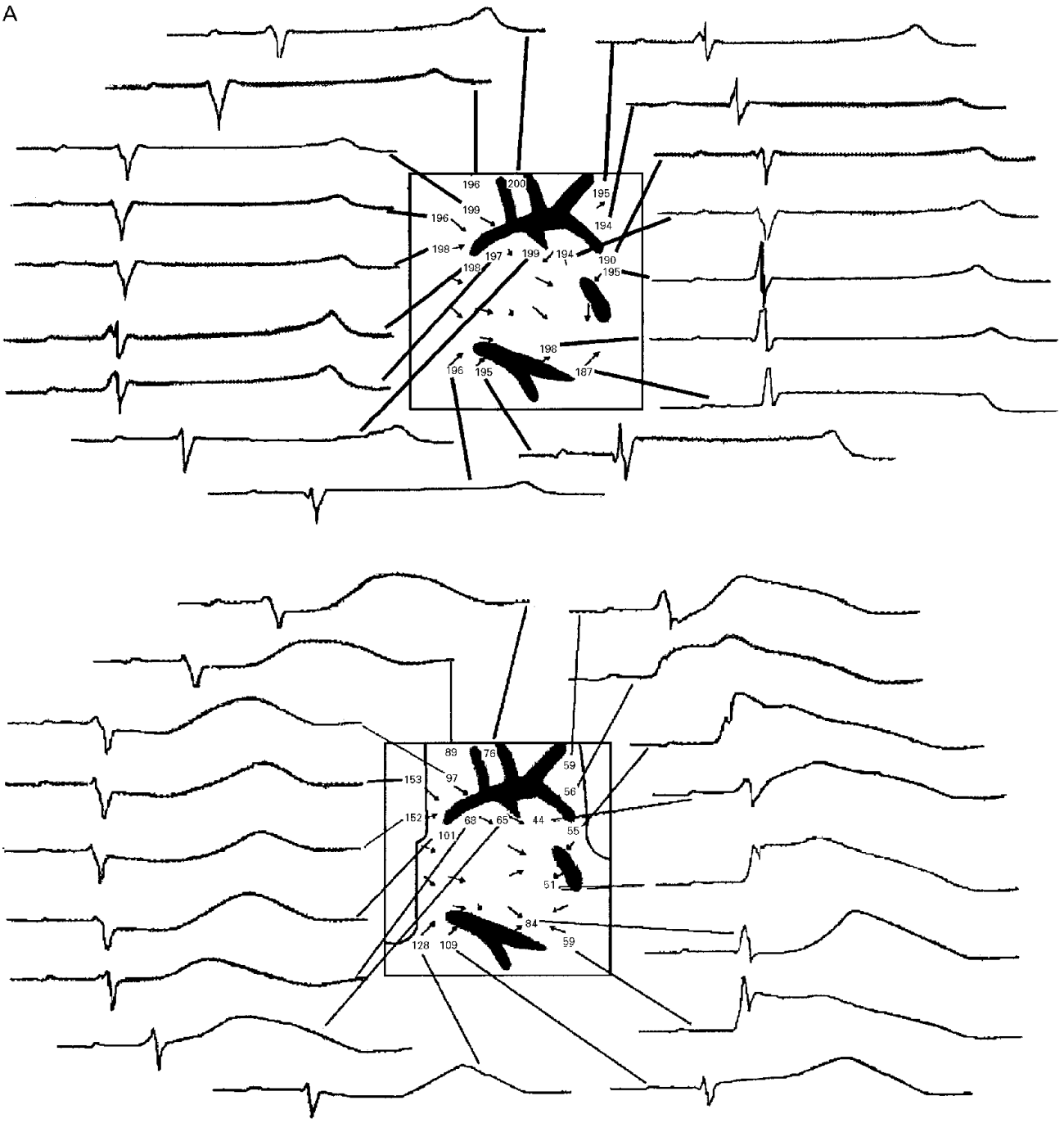

B

\begin{tabular}{|c|c|c|c|c|c|c|c|}
\hline 199 & 196 & 196 & 200 & 195 & 194 & 195 & 192 \\
\hline 199 & 196 & 199 & 198 & 196 & 194 & 194 & 195 \\
\hline 198 & 198 & 198 & 197 & 199 & 197 & 190 & 191 \\
\hline 197 & 196 & 194 & 195 & 194 & 195 & 193 & 194 \\
\hline 196 & 194 & 200 & 200 & 195 & 192 & 193 & 197 \\
\hline 198 & 193 & 195 & 195 & 195 & 189 & 192 & 185 \\
\hline- & 196 & 195 & 199 & 198 & 196 & 187 & 190 \\
\hline 194 & 192 & 194 & 197 & 199 & 196 & 195 & 190 \\
\hline
\end{tabular}

Equilibrium

\begin{tabular}{|c|c|c|c|c|c|c|c|}
\hline 146 & 81 & 89 & 76 & 64 & 54 & 59 & 47 \\
\hline 159 & 153 & 97 & 72 & 67 & 44 & 56 & 52 \\
\hline 161 & 152 & 101 & 68 & 65 & & 55 & 50 \\
\hline 158 & 153 & 110 & 75 & 62 & 5 & 46 & 45 \\
\hline 162 & 147 & 100 & 80 & 58 & 51 & 44 & 40 \\
\hline 153 & 124 & 104 & 85 & 76 & 58 & 45 & 35 \\
\hline- & 128 & 109 & 89 & 84 & 68 & 59 & 44 \\
\hline 152 & 126 & 106 & 98 & 89 & 82 & 58 & 48 \\
\hline
\end{tabular}

10 Minutes regional ischaemia

Figure 2 (A) Graphically reproduced images of the left ventricular wall during control (upper panel) and after 10 minutes of regional ischaemia (lower panel) in a typical experiment. The square represents the shape of the electrode grid, while black areas represent vessel sections. Numbers indicate the activation-recovery interval (ARI) in ms at that particular electrode. Vectors show local velocity and orientation of the propagating wave front at that point. Around the grid, original recordings of the unipolar ECGs of the respective electrode are displayed. The ischaemic border zone is represented as a black line in the lower part of the figure. The formerly homogeneously distributed ARI, which resemble each other closely in morphology, change to heterogeneous ARI with different morphology; the most pronounced shortening is in the direction of the propagating wavefront behind the vessel reflected by a shifted T wave in the ECG to the left and a less pronounced shortening directly in front of the vessel in the direction of the wavefront. Total activation time under control conditions was $5 \mathrm{~ms}$, and after 10 minutes of ischaemia, $11 \mathrm{~ms}$. (B) Left panel: ARI of the left ventricular wall under equilibrium conditions. Right panel: ARI of the left ventricular wall after 10 minutes of regional ischemia. The location of the vessels as seen in the histological image are displayed. 
A
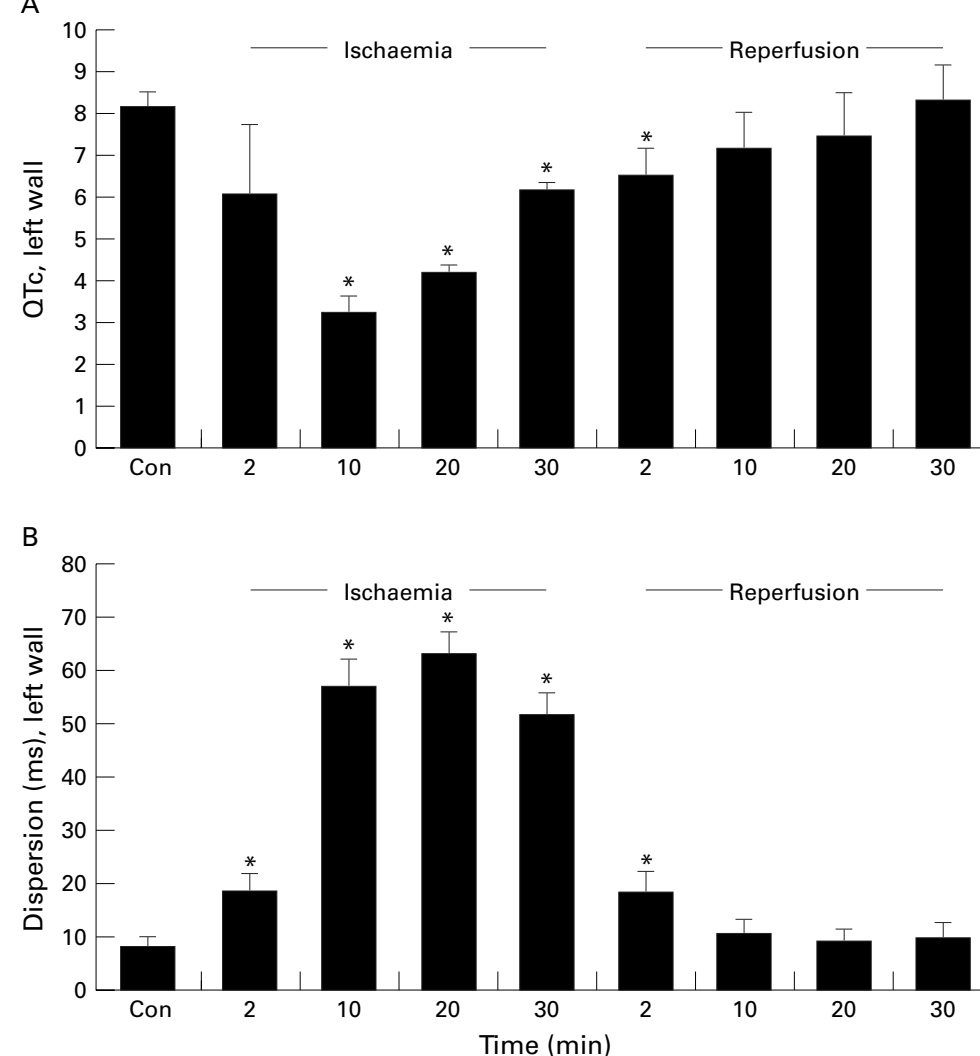

Figure 3 (A) $Q T$, calculated as ARI (activation-recovery interval)/BCL (basic cycle length), of the left ventricular wall; (B) Local dispersion of the left ventricular wall. Values are given as the means of six experiments in the course of ischaemia and reperfusion. Error bars $=S E M .{ }^{\star} p<0.05 v$ values after 60 minutes of equilibration (Con).

experimenter. From these data the activationrecovery interval (ARI, corresponding to the epicardial potential duration) was calculated for all electrodes. ARI were only calculated if the QRS complex showed both a positive and a negative component and if there was a well defined $T$ wave. Other ARI were excluded from the analysis. It has been shown by Millar and coworkers $^{25}$ that changes in the ARI of unipolar epicardial potentials reflect changes in local refractory period. In addition the Qtc time was calculated as Qtc $=\mathrm{ARI} / \mathrm{BCL}$ (basic cycle length). The standard deviation of the ARI, designated as ARI dispersion, was determined for each electrode grid (front, left, right and back). In order to allow a quantitative and comparative description of the activation process, an activation vector was calculated for each electrode from the activation times and the locations of the surrounding electrodes, which were activated after the central electrode. ${ }^{22-24}$ These vectors gave direction and apparent velocity of local activation. We also calculated the ST segment elevation by summing all the deviations from the isoelectric level at the 50\% mean ARI time point. An increase in ST segment elevation indicates an efflux of positively charged ions during the action potential and can be used to determine the ischaemic area. $^{26}$ The total activation time of each electrode grid was determined as the latency between the activation time point of the first and the last electrode activated on that grid.
HISTOLOGY

After the experiment the positions of the electrode grids were marked by injection of ink and afterwards the heart was fixed and embedded in Technovit 7100. Acrylate sections $5 \mu \mathrm{m}$ thick were cut and stained with haematoxylin and eosin. All visual fields of the ink marked tissue were scanned photographically and a histological image of the complete area was composed (yielding a $1 \mathrm{~m}^{2}$ image). Vector fields were printed on transparencies at the same scale as the histological photographs, oriented using the ink marks and overlayed on the histological image. This microscopic image with the projection of the electrode pattern and vectors was reproduced photographically. At each electrode the longitudinal fibre axis was determined and the angle between the local vector and the underlying fibre axis of the tissue could be measured, as shown schematically in fig 1 . The cut depth used for graphic reproduction of the tissue was located 100 to $300 \mu \mathrm{m}$ deep in the epicardium.

\section{REAGENTS}

All reagents used in this study were purchased from Sigma (St Louis, Missouri, USA) and were of analytical grade unless otherwise indicated. Heparin was purchased from Serva (Heidelberg, Germany). Technovit 7100 was obtained from Heraeus-Kulzer (Wehrheim, Germany). Formaldehyde (37\%) was purchased from Baker (Deventer, The Netherlands).

\section{STATISTICS}

All values are given as means (SEM) of seven experiments. Statistical analysis was performed using analysis of variance with subsequent Student $t$ test at a level of significance of $\mathrm{p}<0.05$.

\section{Results}

Occlusion of the left anterior descending coronary artery resulted in a reduction of coronary flow from 21.5 (1.8) $\mathrm{ml} / \mathrm{min}$ during the equilibration period to $11.2(0.6) \mathrm{ml} / \mathrm{min}$ after 30 minutes of ischaemia (54.2(5.4)\%, $\mathrm{p}=0.02)$. Ischemia reduced left ventricular pressure from 81.3 (3.7) to $42(1.3) \mathrm{mm} \mathrm{Hg}$, a decrease of $51.7(3.1) \%(p=0.02)$. The ST segment elevation and the extent of ischaemia (as indicated by the ST segment elevation) were increased by ischaemia: ST-as the integral of the local ST elevations at each site-was increased from 33.2 (10.7) to 284 (42) arbitrary units $(\mathrm{au})(\mathrm{p}=0.01)$ and the area of ST elevation was found to be 39.31 (12.75) $\mathrm{mm}^{2}$ after 30 minutes of ischaemia.

The total activation time was significantly prolonged, from 9.29 (1.55) to 23.92 (5.52) $\mathrm{ms}$ after 30 minutes of ischaemia, and recovered during reperfusion to 11.38 (3.36) $\mathrm{ms}$ within 30 minutes of reperfusion. In addition, the ARI was shortened from 159.7 (11.2) to $129.2(13.2) \mathrm{ms}(\mathrm{p}=0.03)$ up to the end of the ischaemic period. The dispersionthat is, the standard deviation of the epicardial ARI-increased from $7.6(2.2)$ to 50.9 (4.5) $\mathrm{ms}(\mathrm{p}=0.02)$. The local dispersion was also increased. 


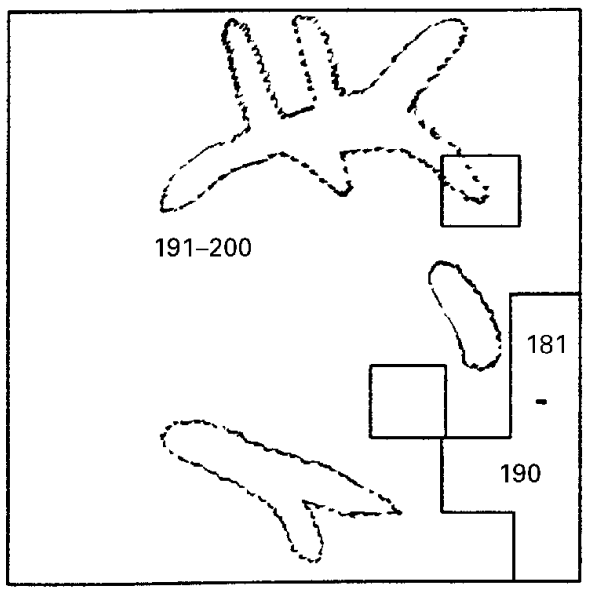

Equilibration

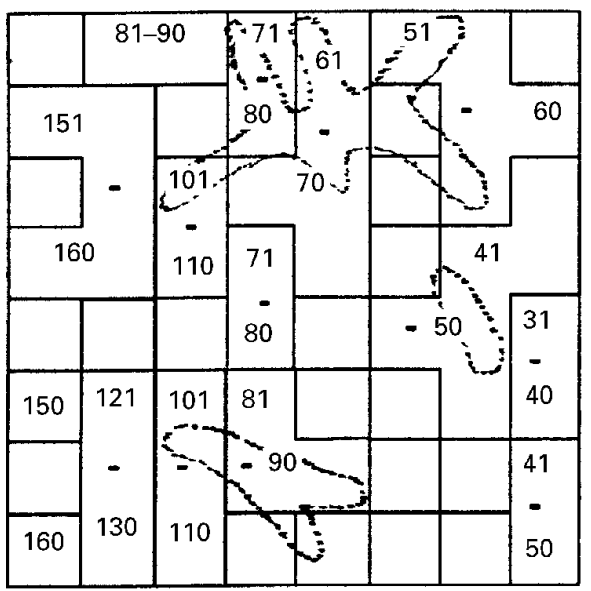

$10 \mathrm{Min}$ ischaemia

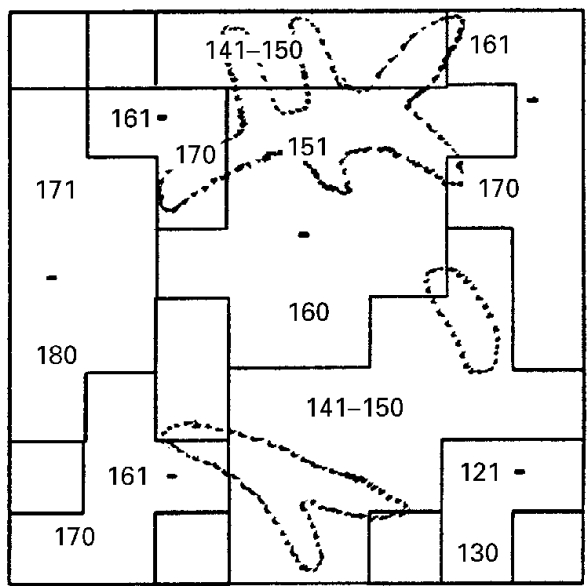

2 Min ischaemia

Figure 4 Activation-recovery interval (ARI) isochrones of a typical experiment. The isochrones indicate areas of ARI within the same range as given by the numbers. The graph shows the situation for the equilibration period, at two and 10 minutes of regional ischaemia, and after 30 minutes of reperfusion.

Figure 2A shows the schematically reproduced tissue of the left ventricle under the electrode grid (square) in a typical experiment. The upper part of the figure represents the situation under control conditions while the lower shows the situation after 10 minutes of regional ischaemia. The ARI are homogeneously distributed from $190 \mathrm{~ms}$ (down right) to $200 \mathrm{~ms}$ (top) around the upper vessel section during equilibration, corresponding to a mean local ARI of $196.3 \mathrm{~ms}$ and a local dispersion of $2.68 \mathrm{~ms}$. The main orientation of the vectors is from the upper left to bottom right. After 10 minutes of regional ischaemia of the left ventricular wall the ARI are generally shortened, as can usually be observed in ischaemic tissue. However, it can be seen that the ARI is heterogeneously shortened.

In general, we found that ARI in front of inexcitable zones were shortened by $50-60 \%$, while behind these zones (seen in the direction of propagation) the shortening was more pronounced $(60-80 \%)$ regardless of whether the local inexcitable zone was located at the border or within the centre of the ischaemic zone. While the ARI in the direction of the propagating impulse at some distance in front of the vessel ranged between 76 and $89 \mathrm{~ms}$, the ARI directly in front of the vessel and in the direction of the wavefront were less shortened and varied between 97 and 153 and 97 and $152 \mathrm{~ms}$, respectively. Behind the vessel-seen in the direction of the propagating impulsethe shortening was even more pronounced, with ARI ranging between 44 and $68 \mathrm{~ms}$. An intermediate ARI of $101 \mathrm{~ms}$ could be seen to the left of the vessel. The mean local ARI of the 12 electrodes considered under these conditions was $84.6 \mathrm{~ms}$, and the local dispersion increased to $34.6 \mathrm{~ms}$, corresponding to a nearly 13-fold increase in ARI dispersion. Both ARI and $\mathrm{QT}_{\mathrm{c}}$ were shortened in the course of ischaemia and recovered completely during reperfusion, as shown in fig $2 \mathrm{~B}$ and fig 3 . The rise in ARI dispersion starts shortly after the occlusion of the coronary artery (two minutes of ischaemia) and reaches its maximum after 10 minutes of ischaemia (fig 3B). It decreases slightly until the end of the ischaemic period. 


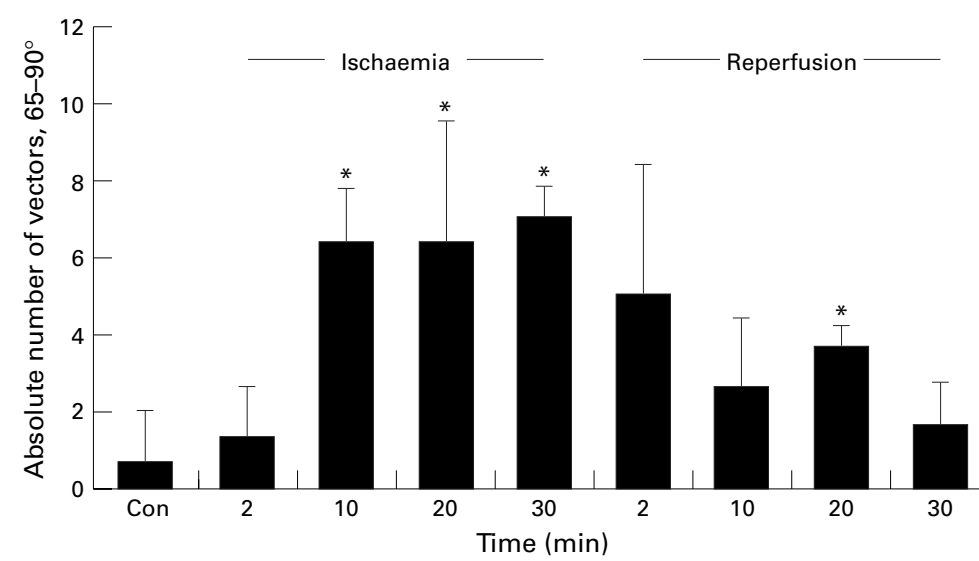

Figure 5 Absolute number of vectors that deviate from the fibre axis between $65^{\circ}$ and $90^{\circ}$ during the course of the experiment. All values are given as means of six experiments. Error bars $=S E M .{ }^{\star} p<0.05 v$ control (that is, 60 minutes at equilibrium).

After 30 minutes of reperfusion we found a nearly full recovery of the ARI and its dispersion. We observed similar changes in the dispersion of the repolarisation time points as well: control, $2.80 \mathrm{~ms}$; two minutes of ischaemia, $18.11 \mathrm{~ms} ; 10$ minutes of ischaemia, $40.5 \mathrm{~ms}$; two minutes of reperfusion, $10.8 \mathrm{~ms}$; and 30 minutes of reperfusion, $5.72 \mathrm{~ms}$. To illustrate the rise of ARI dispersion in the course of regional ischaemia, isochrones were constructed (fig 4). These isochrones encircle areas of ARI within a $10 \mathrm{~ms}$ range. In addition, we found dramatic changes in the activation pattern. The number of vectors with a deviation in the range of $65-90^{\circ}$ from the fibre axis - that is, with propagation transverse to the fibre axis-increased continuously during ischaemia ( $p=0.03$ ) (fig 5) and recovered slowly during reperfusion: thus during ischaemia there was a significant transverse spread of activation.

\section{Discussion}

Since an increase in dispersion is commonly believed to play an important role in the genesis of cardiac arrhythmias ${ }^{102-32}$ and since from a theoretical point of view this dispersion might be enhanced at local tissue non-uniformities, we tried to determine whether tissue inhomogeneities such as fat, connective tissue, or vessels may enhance dispersion, especially during an ischaemic period. The main outcome of this study was the finding that dispersion of ARI is enhanced at sites of tissue inhomogeneity during ischaemia, but not-or only slightly-during a normoxic control period. In addition, activation was slowed during ischaemia (that is, total activation time was increased). Moreover, the total number of vectors deviating more than $65^{\circ}$ from the fibre axis increased during ischaemia, indicating a transverse shift of the spread of activation with respect to the histological fibre axis. It is supposed that this transverse propagation is in part caused by the gap junctional uncoupling due to ischaemia described by Tan et al. ${ }^{12}$ Generally, the longitudinal propagation of activation is more sensitive to reduced sodium channel availability, as shown by the experiments of Spach and Dolber, ${ }^{33}$ whereas transverse propa- gation is more sensitive to gap junctional uncoupling, as demonstrated by Delmar et al. ${ }^{34}$ However, it is not certain whether in a complex situation like ischaemia (with both gap junctional uncoupling and reduced sodium channel availability owing to depolarisation) propagation will fail in a longitudinal or a transverse direction. This probably depends on the degree of both uncoupling and depolarisation and may change in the course of ischaemia. In addition, geometric features such as the number of gap junction channels at the intercalated disk in comparison with the number of gap junctions at the lateral cell border will affect the changes in propagation. However, these geometrical features of the tissue show some species variability.

Reduction in sodium channel availability will slow the impulse propagation ${ }^{35}$ and it can be assumed that this will be enhanced by a concomitant uncoupling of the gap junctions, which are predominantly located at the intercalated disc. The ischaemia induced depolarisation will reduce sodium channel availability and will thus decrease conduction velocity (as indicated by the prolongation of the total activation time), which will result in transverse propagation since the longitudinal conduction depends mainly on sodium channel availability.

With respect to the changes in dispersion, it should be borne in mind that although changes in ARI reflect alterations in local refractory periods, ${ }^{25}$ in the acutely ischaemic myocardium this variable can also be influenced by other factors - such as, for example, the rise in extracellular potassium concentration. However, Gettes and coworkers recently showed in a very elegant study that ARI from unipolar recordings accurately reflect local action potential duration in ischaemic myocardium, ${ }^{36}$ if care is taken that only those potentials with a QRS complex with positive and negative component and a well defined $\mathrm{T}$ wave are evaluated. The changes in ARI cannot be equated with the postrepolarisation refractory period, but the enhanced ARI interval dispersion may also reflect an enhanced dispersion of this variable.

We propose the following mechanism for the observed enhancement of dispersion at tissue inhomogeneities during slowed conduction: if an activation wavefront approaches an inexcitable zone-for example vessels, connective tissue, or fat - it is only possible to have lateral activation, that is, current flows from the excited zone only to the lateral regions and not to the tissue behind, because this is nearly equipotential and is not in the inexcitable zone. However, behind the inexcitable zone activation propagates in three directions - that is, activation current flows to three sites and to a larger area, and thereby the activated cells behind the inexcitable zone may be exposed to a higher electrical burden and thus have shorter potentials than those in front of the inexcitable zone. If in addition the propagation velocity is reduced - for example, in the course of ischaemia, this allows a longer time for the current to flow to the small area before or the large area behind the hindrance, thereby 
enhancing these differences in action potential duration.

What does this mean for cardiology? An increase in dispersion is commonly believed to play an important role in the genesis of arrhythmia according to Spach et al. ${ }^{37}$ This may cause unidirectional block and self sustaining reentry resulting in sustained ventricular fibrillation, ${ }^{11}$ or result in a leading circle. ${ }^{38}$ Slowing of conduction has also long been considered a risk factor for the initiation of arrhythmias. Thus our finding of an enhanced dispersion at sites of histological inhomogeneity during ischaemia may help to explain the enhanced incidence of arrhythmia observed in hearts with histological irregularities, with enhanced histological irregularities, for example in old age, after an infarct or in multiinfarcted hearts, or after myocarditis.

We thank the Deutsche Forschungsgemeinschaft (DFG) for support of this study with grant Dh-3/1-4).

1 Hirche HJ, Franz C, Bös L, et al. Myocardial extracellular $\mathrm{K}^{+}$ and $\mathrm{H}^{+}$increase and noradrenaline release as possible cause of early arrhythmias following acute coronary artery occluof early arrhythmias following acute coronary a
sion in pigs. $\mathcal{F}$ Mol Cell Cardiol 1980;12:579-93.

2 Hill JL, Gettes LS. Effect of acute coronary artery occlusion on local myocardial potassium activity in swine. Circulation on local myocardial

3 Coronel R, Fiolet JWT, Wilms-Schopman FJG, et al. Distribution of extracellular potassium and its relation to electrophysiological changes during acute myocardial ischemia in the isolated perfused porcine heart. Circulation 1988;77: $1125-38$

4 Marban E, Robinson SW, Wier WG. Mechanisms of arrhythmogenic delayed and early afterdepolarisations in ferret ventricular muscle. $\mathcal{F}$ Clin Invest 1986;78:1185-92.

5 Janse MJ, van Capelle FJL, Mosink H, et al. Flow of "injury current" and patterns of excitation during early ventricular arrhythmias in acute regional myocardial ischemia in isolated porcine and canine hearts. Circ Res 1980;47(2): 151-65.

6 Woitczak J. Contractures and increase in longitudinal resistance of cow ventricular muscle induced by hypoxia. Circ Res 1979;44:88-95.

7 Hiramatsu Y, Buchanan JW, Knisley SB, et al. Ratedependent effects of hypoxia on longitudinal resistance in dependent effects of hypoxia on longitudinal resistance

guinea pig papillary muscles. Circ Res 1988;63:923-9.
8 Massey KD, Minnich BN, Burt JM. Arachidonic acid and lipoxygenase metabolites uncouple neonatal rat cardiac lipoxygenase metabolites uncouple neonatal
myocytes. Am $\mathcal{F}$ Physiol 1992;236:C494-501.

9 Saffitz JE, Corr PB, Sobel BE. Arrhythmogenesis and ventricular dysfunction after myocardial infarction-is anomalous cellular coupling the elusive link? Circulation 1993;87:1742-5.

10 Spach MS, Dolber PC, Heidlage JF. Interaction of inhomogeneities of repolarization with anisotropic propagation in dog atria. A mechanism for both preventing and initiating reentry. Circ Re. 1989;65:1612-31.

11 Spach MS, Josephson ME. Initiating reentry. The role of nonuniform anisotropy in small circuits. $\mathcal{F}$ Cardiovasc Electrophysiol 1994;5:182-209.

12 Tan HL, Mazon P, Verberne HJ, et al. Ischaemic preconditioning delays ischaemia induced cellular uncoupling in rabbit myocardium by activation of ATP sensitive potasrabbit myocardium by activation of ATP sensi
sium channels. Cardiovasc Res 1993;27:644-51.

13 Steenbergen C, Murphy E, Watts JA, et al. Correlation between cytosolic free calcium, contracture, ATP, and irrebetween cytosolic free calcium, contracture, ATP, and irreversible ischemic

14 Marban E, Kitakaze M, Koretsune Y, et al. Quantification of $\left[\mathrm{Ca}^{2+}\right]_{\mathrm{i}}$ in perfused hearts: Critical evaluation of the 5F-BAPTA and nuclear magnetic resonance method as applied to the study of ischemia and reperfusion. Circ Res 1990;66:1255-67.

15 Yan GX, Kleber AG. Changes in extracellular and intracellular $\mathrm{pH}$ in ischemic rabbit papillary muscle. Circ Res 1992;71:460-70.
16 Vary TC, Angelakos ET, Schaffer CW. Relationship between adenine nucleotide metabolism and irreversible schemic tissue damage in isolated perfused rat heart. Circ Res 1979;45:218-25.

17 Bastide B, Herve JC, Deleze J. The uncoupling effect of diacylglycerol on gap junctional communication of mammalian heart cells is independent of protein kinase C. Exp Cell Res 1994;214:519-27.

18 Yamada KA, McHowat J, Yan GX, et al. Cellular uncoupling induced by accumulation of long-chain acylcarnitine during ischemia. Circ Res 1994;74:83-95.

19 Lesh MD, Pring M, Spear JF. Cellular uncoupling can unmask dispersion of action activation-recovery interval in ventricular myocardium. A computer simulation study. Circ Res 1989;65:1426-40.

20 Müller A, Dhein S. Sodium channel blockade enhances dispersion of the cardiac action activation-recovery interval. A computer simulation study. Basic Res Cardiol 1993;88:1115.

21 Müller A, Dhein S. Initiation of reentry in a 2-dimensional model of cardiac electrical activity. Pflügers Arch 1995; 429(suppl):R62.

22 Dhein S, Müller A, Klaus W. Prearrhythmia: changes preceding arrhythmia, new aspects by epicardial mapping. Basic Res Cardiol 1990a;85:285-96.

23 Dhein S, Müller A, Gerwin R, et al. Comparative study on the proarrhythmic effects of some class I antiarrhythmic agents. Circulation 1993;87:617-31.

24 Dhein S, Rutten P, Klaus W. A new method for analysing the geometry and timecourse of epicardial potential spreading. Int f Biomed Comput 1988;23:201-3.

25 Millar CK, Kralios FA, Lux RL. Correlation between refractory periods and activation recovery intervals from electrograms: effects of rate and adrenergic interventions. Circulation 1985;72:1372-9.

26 Dhein S, Müller A, Klaus W. Nifedipine antagonizes ouabain-induced ST-segment changes and derangement of epicardial activation pattern in isolated rabbit hearts. Int $\mathcal{F}$ Cardiol 1990;29:163-72.

27 Durrer D, van der Tweel LH. Spread of activation in the left ventricular wall of the dog. II. Activation conditions of the epicardial surface. Am Heart f 1954;47:192-203.

28 Han J, Moe GK. Nonuniform recovery of excitability in ventricular muscle. Circ Res 1964;16:46-60.

29 Merx W, Yonn MS, Han J. The role of disparity in conduction and recovery time on ventricular vulnerability to fibrillation. Am Heart f 1977;94:603-10.

30 Kleber AG, Janse MJ, van Capelle FJL, et al. Mechanism and time course of S-T and T-Q-segment changes during acute regional myocardial ischemia in the pig heart determined by extracellular and intracellular recordings. Circ Res 1978;42:603-13.

31 Kuo CS, Munakata K, Reddy CP, et al. Characteristics and possible mechanisms of ventricular arrhythmias dependant on the dispersion of action activation-recovery interval. Circulation 1983;67:1356-67.

32 Tan RC, Joyner RW. Electrotonic influences on action potentials from isolated ventricular cells. Circ Res 1990;67: 1071-81.

33 Spach MS, Dolber PC. Discontinuous anisotropic propagation. In: Rosen MR, Janse MJ, Wit AL, eds. Cardiac electrophysiology. Mount Kisco, NY: Futura, 1990:517-34

34 Delmar M, Michaels DC, Johnson T, et al. Effects of increasing intercellular resistance on transverse and longitudinal propagation in sheep epicardial muscle. Circ Res 1987;60:780-5.

35 Buchanan JW, Saito T, Gettes LS. The effects of antiarrhythmic drugs, stimulation frequency and potassium induced resting membrane potential changes on conduction velocity and $\mathrm{dV} / \mathrm{dt}$ in guinea pig myocardium. Circ Res 1985;56:696-703.

36 Gettes LS, Engle CL, Sherman ZC, et al. Activation recovery intervals accurately reflect action potential duration during acute ischemia. Circulation 1997;96(supp1 I):I-59.

37 Spach MS, Dolber PC, Heidlage JF. Influence of the passive anisotropic properties on directional differences in propagation following modification of the sodium conductance in human atrial muscle. A model of reentry based on anisotropic discontinuous propagation. Circ Res 1988;62:81132 .

38 Allessie MA, Bonke FIM, Schopmann GJG. Circus movement in rabbit atrial muscle as a mechanism of tachycardia. III. The "leading circle" concept: a new model of circus movement in cardiac tissue without the involvement of an anatomical obstacle. Circ Res 1977;41:9-18. 